\title{
Review Article \\ Obesity as a Major Risk Factor for Cancer
}

\author{
Giovanni De Pergola and Franco Silvestris \\ Department of Biomedical Sciences and Human Oncology, Section of Internal Medicine and Oncology, \\ University of Bari "Aldo Moro", School of Medicine, Policlinico, Piazza Giulio Cesare 11, 70124 Bari, Italy \\ Correspondence should be addressed to Giovanni De Pergola; gdepergola@libero.it
}

Received 18 December 2012; Revised 17 July 2013; Accepted 25 July 2013

Academic Editor: Francesco Saverio Papadia

Copyright (C) 2013 G. De Pergola and F. Silvestris. This is an open access article distributed under the Creative Commons Attribution License, which permits unrestricted use, distribution, and reproduction in any medium, provided the original work is properly cited.

\begin{abstract}
The number of cancer cases caused by being obese is estimated to be $20 \%$ with the increased risk of malignancies being influenced by diet, weight change, and body fat distribution together with physical activity. Reports from the International Agency for Research into Cancer and the World Cancer Research Fund (WCRF) have shown that the strongest evidence exists for an association of obesity with the following cancer types: endometrial, esophageal adenocarcinoma, colorectal, postmenopausal breast, prostate, and renal, whereas the less common malignancies are leukemia, non-Hodgkin's lymphoma, multiple myeloma, malignant melanoma, and thyroid tumours. To be able to develop novel methods in prevention and treatment, we first must understand the underlying processes which link cancer to obesity. Four main systems have been identified as potential producers of cancer in obesity: insulin, insulin-like growth factor-I, sex steroids, and adipokines. Various novel candidate mechanisms have been proposed: chronic inflammation, oxidative stress, crosstalk between tumour cells and surrounding adipocytes, migrating adipose stromal cells, obesity-induced hypoxia, shared genetic susceptibility, and the functional defeat of immune function. Herein, we review the major pathogenic links between obesity and susceptibility to cancer.
\end{abstract}

\section{Introduction}

Obesity is a serious problem which heightens the risk of several chronic illnesses including cancer development [1-3]. Current recommendations from the Public Health Goals of the World Cancer Research Fund (WCRF) suggest that the median adult BMI should be maintained between 21 and $23 \mathrm{~kg} / \mathrm{m}^{2}$, depending on the normal range for different populations [4].

\section{Obesity and Cancer Risk}

2.1. Epidemiology and General Factors. It has been estimated that about $20 \%$ of all cancers are caused by excess weight [5], and the Million Women Study, the largest study of its kind on women, has shown that approximately half can be attributed to obesity in postmenopausal women [6]. There are many prospective epidemiological studies which have demonstrated a direct association between overweight and cancer, even though obesity alone does not apparently heighten cancer risk in all tissues by the same amount [1-7].
A recent systematic review and meta-analysis of prospective observational studies [3], with 282,000 incident cancer cases and a follow-up greater than 133 million person-years, has demonstrated that the obesity and cancer association is sex specific over a wide range of malignancies, and this remains mostly true for different geographic populations. However, cancer risk in obesity is different between ethnic groups [3] in that African Americans appear rather susceptible to cancer, in contrast to Hispanics who appear to be relatively protected while the association of increased BMI with breast cancer is particularly strong in Asia-Pacific populations [3].

The International Agency for Research into Cancer (IARC) [7] and the World Cancer Research Fund (WRCF) reports [4] showed that common cancers in obese people are predominantly endometrial, esophageal adenocarcinoma, colorectal, postmenopausal breast, prostate, and renal. Less common malignancies associated with obesity are malignant melanoma, thyroid cancers [8], and leukemia, non-Hodgkin's lymphoma, and multiple myeloma [9]. 
The role that obesity plays in carcinogenesis has been brought to the fore by data such as the rapid rise of oesophagus adenocarcinoma over the past 20 years. In fact, whether oesophageal reflux is associated with adenocarcinoma [10], or whether it is favoured by obesity [11], the change in oesophageal cancer morphology from squamous to adenocarcinoma has followed the worldwide rise in obesity. A further example is provided by weight accumulation with age which is also linked to an increase in postmenopausal breast cancer risk in women who do not follow a menopausal hormone therapy regime [12], while cohort studies have shown that breast cancer risk was lowered by $50 \%$ in women who intentionally underwent weight loss higher than $10 \mathrm{~kg}$ after menopause [13]. In addition, the Swedish Obese Subjects (SOS) study, a large prospective study which established that bariatric surgery achieves an average of $20 \mathrm{~kg}$ weight reduction in obese patients with BMI higher than $40 \mathrm{~kg} / \mathrm{m}^{2}$ and that matched the surgery group with untreated morbidly obese women, reported a significant reduction in cancer incidence in association with substantial weight loss on a followup longer than 10 years [14]. Concerning the role of childhood obesity in adult cancer, a study performed in a cohort of 2,347 subjects retrospectively evaluated cancer risk related to age and sex-specific BMI standard deviation scores (SDS) of subjects aged 2-14 [15]. Among these subjects, the risk of cancer in adult life was increased by $9 \%$ per SD increase in childhood BMI [15]. Also, an excess of weight in teenagers was linked to doubling the mortality risk of colon cancer in adulthood [16]. Because of the time lag in cancer development in the presence of adiposity, the typical follow-up is longer than 10 years in cohorts assessing cancer risk, and it is thought that this is an "average" lag period for obesity-related cancer development [17]. However, the lag period may be shorter when hyperoestrogenemia is a predominant mechanism, as it occurs in postmenopausal breast and endometrial cancer.

2.2. Relation with Body Fat Distribution. Apart from BMI, other anthropometric measurements, such as waist-to-hip ratio (WHR) or waist circumference (WC), are currently being investigated as indicators of adiposity and cancer risk. It has been shown that cancers that are associated more with abdominal adiposity than with BMI predominantly include colon, premenopausal breast, endometrium, and oesophageal adenocarcinoma, together with pancreas tumours [18-23]. A recent longitudinal study (median of 5.0 years), performed in participants from the Framingham Heart Study, has shown that visceral adiposity, measured by using multidetector computed tomography, is associated with cancer after adjustment for clinical risk factors and generalized adiposity [24]. As for mortality, a longitudinal study in US women has shown that WC and WHR are strongly and positively associated with cancer mortality, independently of BMI [25].

2.3. Effect of Weight Change. A Canadian case-control study has found that those men whose weight increased by more than $21 \mathrm{~kg}$ after reaching adulthood had a colorectal cancer risk $60 \%$ higher than those with a weight increase of $1-5 \mathrm{~kg}$, and the association was even stronger after excluding those patients with rectal tumours [26]. Endometrial cancer has also seemingly been shown to be directly correlated with weight gain during adulthood among Japanese women [27]. Additionally, diet-induced weight loss reduces colorectal inflammation and cancer-related gene pathways in obese individuals [28].

\section{Obesity and Cancer Mortality}

The American Cancer Society has furnished data which suggest that overweight and obesity are correlated to liver and pancreatic cancers death rates, as are both myeloma and nonHodgkin's lymphoma [1].

Data published over the last 25 years emphasize that obesity is a cause of about $20 \%$ of deaths from cancer in women while the rate is around $14 \%$ in men. These rates are second only to smoking for the number of avoidable cancers [1]. However, these data are possibly underestimated, since average weight has continued to rise in the worldwide population over the same time. Recently, Jaggers et al. found a positive multivariable-adjusted association between cancer mortality and abdominal obesity that increases the risk of cancer mortality up to $24 \%$ [29]. Epidemiological studies have also shown that obesity is a contributing factor in both increased incidence and mortality from cancers of the colon, the endometrium, the kidneys, and the breast (in postmenopausal women) [5, 6]. In the European Union, it has been calculated that not becoming overweight could reduce the annual incidence of colon cancer by up to 21,000 cases and breast cancer by up to 13,000 cases. The higher cancer death rate in the obese also accounts for a decreased survival rate, that may be due to the enhancing effects of obesity on cancer potency and progression. A study on invasive ductal breast cancer in 1,177 women found that those in the highest quartile of BMI developed tumours with a higher histological grade, mitotic cell count, and larger tumour size compared to women in the lower quartiles [30]. Furthermore, by considering solely large tumours up to $2 \mathrm{~cm}$ of diameter, those in the group of the highest BMI quartile largely expressed markers of high proliferation, supporting their fast growth, as compared to those with similar-size tumours in nonobese women [30]. However, it has been reported that chemotherapy dosages are often not adequate in women of excess weight receiving breast cancer treatments when compared to dosages in lean women [31], thus implying that an overall full weightbased adjusted dose would allow better outcomes.

\section{BMI-Cancer Associations and Histological Subtypes}

Several data suggest that the BMI-cancer association is typical of a number of histological subtypes within a site specific cancer. These examples include the following.

(a) A dose-response meta-analysis ( 9 cohorts: 22 casecontrol studies) showed that the BMI-breast cancer association is stronger for oestrogen receptor positive/progesterone receptor-positive $(\mathrm{ER}+\mathrm{PR}+)$ tumours $\left(33 \%\right.$ increase per $5 \mathrm{~kg} / \mathrm{m}^{2}$ increment for postmenopausal breast cancer), while there were no 
significant BMI-cancer associations for ER-PR- and ER+PR-tumours.

(b) Detailed pathological examination of a Swedish population-based prospective cohort (9,685 postmenopausal women not using hormone therapy) showed that the highest quartiles of BMI were associated with ductal type tumours, grade II, low Ki67 index, HER2 negativity, low-cyclin D1 expression, ERa, and PR immune-positivities, but not ERb tumours.

(c) For endometrial cancer, obesity is predominantly a risk factor for type I endometrioid tumours (accounting for $70 \%$ of endometrial cancers) rather than type II.

(d) BMI associations are stronger for the papillary subtype of thyroid carcinoma.

(e) Positive associations exist between BMI and cardias gastric adenocarcinomas, but not noncardia gastric malignancies.

The biological mechanisms responsible for stronger association of BMI with some cancer subtypes are not known, but these information might have implications in determining the patient's risk and the subsequent recommendations.

\section{Biological Mechanisms Linking Abdominal Obesity to Cancer Susceptibility}

In the obese, the mechanisms which may foster or promote cancer occurrence or progression are usually classified as being of two types. They may be universal and direct in nature in that they apply to all or the majority of tumours because they have to do with the hormonal and/or metabolic abnormalities prevalent in obesity. Alternatively, they may be site specific, in which conditions can foster a particular tumour in a particular site because they have to do with the consequential effects of obesity, so leading to complications in specific tissues or organs. There is, for example, a heightened risk of cancer of the gallbladder when gallstones are present and of oesophageal adenocarcinoma as a putative effect of aggravated gastrooesophageal reflux. Also liver cancer can be a consequence of nonalcoholic steatohepatitis [32]. Certainly, various syntheses of mechanisms can operate differently in different people.

5.1. Diet. Both epidemiological studies in people and experiments in animals suggest that alterations in caloric intake or in quality of diet may significantly influence the risk of cancer development and progression. However, the separate effects of diet per se, as well as of positive energy balance, reduced physical activity, and increased adiposity, are not easy to determine. It is well known that caloric excess will lead to increased cancer incidence and that positive energy balance appears to promote cancer cell proliferation and tumour progression. By the same token, limited long-term calorie intake has led to a decrease in cancer incidence and extended longevity in rodents [33]. There are many studies, not withstanding the symptomatic underreporting of surveys, which document the health hazards related to diets typical of the first world, rich in calories, overabundant in alcohol and animal fats, and/or lacking in plant products [34]. By contrast, a defined association between low-cancer risk and a higher intake of fruit and vegetables has been proven. High-fibre cereal consumption has also been linked to a reduced risk of colorectal cancer, although large-scale studies do not demonstrate such a benefit [34]. The negative energy balance may be an important factor in lowering the susceptibility to cancer because of direct or indirect effects on levels of insulin [35], insulin-like growth factor-I (IGF-I) [35], and inflammatory biomarkers [36]. Interestingly, preliminary data demonstrate that a low carbohydrate insulin-inhibiting diet, associated with ketosis, is safe and correlated with stable disease or partial remission in patients with advanced cancer [37]. It is noteworthy that obesity is correlated with low-serum levels of 25- hydroxyvitamin D (25 (OH)D), confirmed by a study showing that low vitamin D levels may be responsible for $20 \%$ of the cancer risk linked to increased BMI [38].

5.2. Physical Activity. For the prevention of the onset of chronic diseases, including cancer, guidelines suggest exercise as part of the daily routine, a brisk walk or walking to work instead of driving, and/or practicing more physical activity at home, such as using stairs or gardening [39]. There have been several studies which show that physical exercise can lower breast cancer risk and that there are benefits both physical and mental for cancer patients [39]. It has also been correlated to a decrease in the risk of both colon and breast cancer development, and probably with a lower risk of endometrial cancer [39].

Regarding possible mechanisms responsible for cancer prevention, regular physical activity helps maintain a healthy body weight, regulates sex hormones, insulin, and prostaglandins and has various beneficial effects on the immune system [40]. The specific dose of exercise needed to optimize primary cancer prevention remains to be elucidated.

5.3. Insulin Resistance. The most popular hypothesis explaining the association between obesity and cancer is that of lower insulin sensitivity. Excess body weight and adiposity are directly correlated with insulin resistance, compensated for by the stimulation of pancreatic insulin secretion, and this commonly results in hyperinsulinemia. Elevated insulin serum levels favour faster growth and increased aggressiveness of colorectal [41, 42], pancreatic [41], liver [41], postmenopausal breast $[41,43,44]$, and endometrial $[41,45]$ cancers. C-peptide is often used, preferably to insulin, as a serum biomarker of pancreatic $\beta$-cell secretory activity because of its longer half-life due to the lack of hepatic extraction, its lower metabolic clearance rate, and its lack of cross-reactivity with insulin antibodies. Several epidemiological cohort studies have indicated a direct correlation between circulating Cpeptide levels and colorectal [46, 47], pancreatic [36, 46], liver [46] endometrial [46, 48], and postmenopausal breast [49] cancers. However, prospective cohort studies have not demonstrated any association between prostate cancer risk and elevated C-peptide levels or high insulin levels, although a more elevated risk of prostate cancer mortality for men with the highest $\mathrm{C}$-peptide levels has been recently reported after 
adjustment for BMI [50]. Although insulin itself does not induce somatic cell mutations, it has anabolic and antiapoptotic effects, and it is thought that only supraphysiological concentrations of insulin have mitogenic effects. Interestingly, cancer cells commonly have the ability to respond to the activation effect of insulin on intracellular transduction pathways, particularly when insulin is highly concentrated, as occurs in obesity. Signalling by the insulin pathway is highly relevant in cancer development as both extracellular-signalregulated kinase (ERK) and phosphatidylinositol-3 kinase (PI-3 K) pathways are triggered by activation of the insulin receptor (IR). The insulin receptor is highly expressed in adipose, muscle, and kidney tissues, while it is overexpressed in breast [51] and prostate [52] cancer cells as well as in most normal and neoplastic haematopoietic cells [51]. It has been hypothesized that the IGF-I receptors are the mediators for the main proliferative effects of insulin as the insulin receptor (IR) and the receptor for IGF-I (IGF-IR) show more than 50\% of overall sequence homology and even $84 \%$ of homology in the tyrosine kinase domain of the $\alpha$-subunit [43] while their ligands, insulin, and IGF-I share $40-50 \%$ homology. Because of this, insulin and IGF-I can interact either with IR or with IGF-IR [45], and both of these receptors have been associated with tumour development.

Regarding increased tumour invasion, biochemical and functional analyses have demonstrated that ligand-induced activation of the IGF-IR kinase results in the loss of epithelial integrity, at the same time promoting cell migration [53]. In addition to the specific receptors IR and IGF-IR, coexpressed in the same cell, a third receptor, a hybrid form (IR-IGFIR) between IR and IGF-IR, can mediate the effects of both insulin and IGF-I as a result of their high homology [46]. Interestingly, over-expression of both IR and IGF-IR in a variety of cancer tissues leads to heterodimerization and the formation of this hybrid receptor in amounts that even exceed IGF-IR content, especially in malignant breast and thyroid tissues [51]. The hybrid receptor in malignant tissues predominantly includes IGF-IR and IR isoform A, which has a higher affinity for binding IGF-I than insulin does and stimulates cell proliferation.

5.4. IGFs and IGF Binding Proteins (and Their Interrelationship with Insulin and Insulin Resistance). The indirect effects of hyperinsulinemia on carcinogenesis are attributable to the action of insulin on circulating endogenous growth factors together with their binding proteins. In hyperinsulinemia, the growth hormone receptor (GHR) is upregulated by the increase of insulin concentrations in portal circulation thus increasing GHR signalling and hepatic IGF-I synthesis [54]. Moreover, insulin decreases the hepatic expression of binding proteins of IGF-I, such as insulin-like growth factor binding proteins-1 and -2 (IGFBP-1, IGFBP-2) [55], thus leading to higher plasma levels and bioavailability of free IGF-I $[2,56]$. In this context, IGFBP-1 inhibits the growth of breast cancer cells in mice with MCF-7 breast cancer xenografts, as well as hepatocellular cancer growth in mice over-expressing IGF-I and IGF-II [54]. IGF-I also exerts proangiogenic effects and induces tumour-related lymphangiogenesis. In fact, IGF-I induces the synthesis of hypoxia-inducible factor-la
(HIF-1a), which together with vascular endothelial growth factor (VEGF) [56] leads to neovascularization and metastases, as shown in a colon cancer model [57]. Moreover, through the inhibition of p53, IGF-I prevents apoptosis [56] and induces metastatic tumour spread, linked to integrins movement to the borders of migrating cells [58]. In addition to insulin and IGF-I, over-expression of IGF-II, a hormone produced in the liver, is also associated with tumour development. IGF-II signalling takes place through IR (specifically the mitogenic IR-A subtype) and IGF-IR. Another factor influencing IGF-I free levels is insulin-like growth factor binding protein-3 (IGFBP-3), which is the most profuse of the IGFBPs. Worthy of note is that visceral fat accumulation leads to lower IGFBP-3 plasma levels [59], and lower IGFBP-3 concentrations lead to higher free IGF-I levels. Moreover, IGFBP-3 exerts direct protective effects against cancer development, by inducing apoptosis through both p53-dependent mechanisms and Bcl-2 family proteins [54]. IGFBP-3 also interacts with the nuclear retinoid X receptor (RXR)-a, altering its binding to the transcription factor Nur 77 and activating the apoptosis cascade [54].

5.5. Sex Hormones. In women, BMI has been regularly correlated with breast, endometrium, and other cancers linked to hormones, since an increase in circulating sex steroids from weight excess is a well-known mechanism implicated in cancer development. Data from epidemiological studies demonstrate that the postmenopausal risk of breast cancer increases in women with heightened levels of circulating sex steroids, including dehydroepiandrosterone (DHEA), its sulfate (DHEAS), $\triangle 4$-androstenedione, testosterone, estrone, and total estradiol, and low levels of sex hormone binding globulin (SHBG). Endogenous sex hormones are active in tumour cell growth and directly mediate the effect of obesity on cancer, in particular in tumours of the breast [60-62] and the endometrium [63]. There is evidence that estrogens are mitogenic [64], regulate the expression of insulin receptor substrate-1 (IRS-1) in the breast [65], and induce free radical-mediated DNA damage, genetic instability, and gene mutations in cells. The Million Women Study [6] and previous studies have shown that in premenopausal women breast cancer risk decreases with increasing BMI, while it increases with BMI in postmenopausal women who do not practice hormone replacement therapy, possibly owing to heightened levels female sex hormones in circulation, caused by increased estradiol creation from androgenic precursors in adipose tissue through an increase in activity of the aromatase enzyme. In endometrial cancer, heightened concentrations of plasma estradiol and estrone are correlated with increased cancer risk in postmenopausal obese women [66] and elevations of estradiol increase endometrial cell proliferation while inhibiting apoptosis and at the same time encouraging IGF-I synthesis in endometrial tissue [2]. At variance with postmenopausal women, in premenopausal women a deficiency of progesterone is likely to have more influence than any excess of estrogen. As far the role of androgens is concerned, anthropometric parameters (BMI and waist circumference) and testosterone levels are inversely correlated in men [67] and directly associated in women [62]. 
The EPIC and other epidemiological studies have demonstrated that heightened plasma levels of androgens are associated with a more elevated risk of breast cancer in women both pre- and postmenopausal, therefore suggesting that androgens may also be considered as links between obesity and breast cancer [60-62]. Elevated plasma androstenedione and testosterone levels may also correlate with endometrial cancer risk in both pre- and postmenopausal women [65].

5.6. Adipose Tissue and Adipokine Production. Much metabolic and endocrine activities take place in adipose tissue. In obesity, there is oversecretion of deleterious proinflammatory molecules like interleukin-6 (IL-6), tumour necrosis factor- $\alpha$ (TNF- $\alpha$ ), leptin, resistin, retinol binding protein4 , plasminogen activator inhibitor-1 (PAI-1), hepatic growth factor (HGF), and so forth and hyposecretion of beneficial adipokines such as adiponectin and visfatin [68]. These adipokines can be secreted from both adipocytes and stromal-vascular cells, and most of these substances are actually from macrophages and other immune cells (T-cells, etc.) that infiltrate adipose tissues in obese patients [69]. Interestingly, human adipose tissue macrophages resemble human tumor-associated macrophages and increase the expression of FAS in cancer cells, thus playing a critical role in cancer cell survival [70].

Adiponectin and leptin have been subject to much study in cancer development as they are the most abundant adipokines. Higher leptin levels can be a factor for fostering cancer development and progression since it is now known to be mitogenic, proinflammatory, antiapoptotic, and proangiogenic [71]. Most experimental studies on leptin have been on breast cancer, and they have shown that: (1) leptin upregulates oestrogen/ER signalling; (2) leptin intensifies the expression of aromatase and, therefore, oestrogen production [72]; and (3) leptin receptors are located in both normal and malignant breast epithelial cells. However, while breast tumour cells proliferate after leptin stimulation [73], transgenic mice with no leptin or its receptor have been shown to be resistant to tumour formation [74]. The idea has been put forward that leptin regulates hepatocellular carcinoma development through the modulation of human telomerase reverse transcriptase [75]. There are four splice variants of the leptin receptor; however, only $\mathrm{LRb}$ has a long enough intracellular domain to allow full signal-transducing capabilities. LRb activates the PI3 kinase, MAPK (mitogen-activated protein kinase), and STAT (signal transducer and activator transcription) signalling pathways, which are vital for cell survival, proliferation and differentiation [76], and c-fos gene transcription. Interestingly, isoforms may also have differential roles in ER-positive and ER-negative breast cancer cells [71].

Blocking cancer-stromal cell communication may well be a strategy to target breast cancer therapy, and, interestingly, it has been shown that leptin drives tumour progression by a bidirectional crosstalk between breast cancer cells and cancer-associated fibroblasts [77].

Adiponectin, being the most abundant adipokine, is particularly interesting because it enhances insulin sensitivity, and its circulating levels are inversely related to cancer occurrence and cancer stage [78]. However, adiponectin serum levels correlate negatively with BMI and even more negatively with visceral adiposity [78] while insulin and estrogens may suppress its secretion [79]. Adiponectin has a protective role in carcinogenesis, indirectly by acting against the development of insulin resistance [43] and directly, as in vitro and in vivo studies have shown, by activating the AMPK pathway [43] and inactivating MAPK kinases 1 and 3 and ERK1 and ERK2 [80]. Moreover, adiponectin stimulates apoptosis through the induction of p53 and Bax expression and the decrease of Bcl-2 expression [80], inhibits angiogenesis and cell migration [81], exerts an anti-inflammatory role, and induces downregulation of vascular adhesion molecules, thus reducing tumour spread [82]. Last, adiponectin prevents the interaction of various growth factors with their receptors [83]. Adiponectin levels have been studied in relation to the risk of several cancer types. In breast cancer, two casecontrol studies showed inverse correlations, one study in preand postmenopausal women [84] and the other one only in postmenopausal cases [85]. Breast cancer risk was much lower in postmenopausal women with elevated adiponectin levels in a large prospective case-control study in two North American cohorts [86]. For endometrial cancer, two casecontrol studies have reported significant inverse correlations which seem stronger in younger women [87] while a prospective case-control study, nested within the EPIC, found a lower risk for women in the highest versus lowest quartiles of adiponectin after adjustment for BMI [88]. The Health Professionals Follow-up Study reported that low adiponectin blood levels was a risk factor for colorectal cancer in men [89], but this finding has not been confirmed in other prospective studies. There are no clear-cut results available for other cancers such as pancreas adenocarcinoma or prostate cancer in nonsmoker populations. Several studies have suggested that the activation of peroxisome proliferator-activated receptor- $\gamma$ (PPAR- $\gamma$ ) may be involved in cancer development in obesity, since it inhibits cell proliferation and induces apoptosis in vitro, possibly by antagonizing the activities of NF- $\kappa \mathrm{B}[90]$.

Obesity has several features for altered PPAR- $\gamma$ function, which include an increased production of PAI-1, and there are diverse studies which show that PAI-1 promotes tumour growth through the inhibition of apoptosis. It is also an indicator of poor prognosis for several cancers such as breast cancer [91]. PAI-1 also seems to be involved in angiogenesis, enhanced cell adhesion, and increased migration [91]. Furthermore, in breast cancer, PAI-1 is implicated in inflammatory response, neutrophil recruitment, and proliferation of smooth muscle cells. PPAR- $\gamma$ dysregulation and PAI-1 overproduction in adipose tissue of obese women may change the breast microenvironment and facilitate local cancer development and/or metastasis [91]. Maury et al. have identified other adipokines that are oversecreted by omental adipose tissue (AT) in obesity and which positively correlate with BMI, insulin resistance, and hypoadiponectinemia [92]. In particular, the circulating concentrations of adipokines such as growth-related oncogene factor $\alpha$ (GRO- $\alpha$ ), tissue inhibitor of metalloproteinases-1 (TIMP-1), and thrombopoietin (TPO) are increased in severely obese women $[92,93]$. Therefore, high levels of these molecules are suspected to promote cancerogenesis especially as GRO- $\alpha$ is involved 
in inflammation, angiogenesis, and tumorigenesis [94], and high levels of GRO- $\alpha$ and TIMP-1 may play a role by enhancing cell proliferation, antiapoptotic activity, and/or angiogenesis [94], while TPO stimulates the proliferation and differentiation of megakaryocytes [95].

5.7. Chronic Inflammation and Nuclear Factor- $\kappa B$ System. Obesity is correlated to chronic inflammatory response, with abnormally high cytokine production and the activation of proinflammatory signalling pathways. The subclinical low-grade chronic inflammatory state in obesity might be important in the initiation and promotion of cancer cells [2]. Prospective studies have reported that an increase in circulating C-reactive protein (CRP) is associated with increased risk of colorectal cancer, and, in particular, it has been shown that genetic variation in the CRP gene influences risk of both colon and rectal cancer developments [96]. A casecontrol study has also provided epidemiological evidence that endometrial carcinogenesis may be promoted by an inflammatory milieu [97]. Of the two different pathways which link inflammation with tumorigenesis, one is an extrinsic pathway mediated by chronic inflammation (inflammation-induced cancer) and the other an intrinsic pathway where genetic alterations, without underlying inflammation, originate a tumor-driven host immune response creating a microenvironment made up of inflammatory cells (cancer-induced inflammation) [98]. Both intrinsic and extrinsic pathways link inflammation and cancer by a number of oncogenes (ras/raf, ReIB, nuclear oncogenes, oncosuppressors, etc.) [98, 99]. Interestingly, abundant literature suggests that energy accumulation plays an important part in the origin of chronic inflammation [100], and, in fact, caloric restriction induces low inflammation. Recent evidence gives support to the idea that the inflammatory response is to increase energy expenditure through feedback to compensate for the excess of energy in obesity, and if this feedback system is not efficient (inflammation resistance), energy expenditure is lowered and energy is conserved which then leads to obesity [100].

5.8. Crosstalk between Tumour Cells and Surrounding Adipocytes and Migrating Adipose Stromal Cells. Tumour progression is now seen as resulting from the crosstalk between tumour cells and the "normal" cells which surround them. In breast cancer, mature adipocytes are part of the breast cancer tissue and thus contribute to cancer progression as highly specialized endocrine cells capable of modifying breast cancer cell behaviour. It has been proposed that evolving bidirectional crosstalk is functional between breast cancer cells and tumour-surrounding adipocytes, and that the tumour-modified adipocytes are actively involved in tumour progression [101]. Tumour vasculogenesis is also essential in cancer development, and a possible source of progenitor cells for new vessels could be mesenchymal stromal cells (MSCs) which differentiate into different phenotypes, and which, though at low plasma concentrations, can be attracted by pathological signals such as hypoxia or inflammation to specific tumour sites where they promote angiogenesis, driving tumour progression, and become tumour stromal cells. Other than from bone marrow, MSCs may arise from white adipose tissue (WAT) and other sources [102]. It has been suggested that a higher frequency of MSCs in peripheral blood may well be a diagnostic marker for colorectal cancer [103], and changes in circulating MSCs dependent on BMI, possibly mobilized from white adipose tissue, may be addressed to tumors, representing a link between obesity and cancer progression.

5.9. Adipose Tissue and Vascular Growth Factors. Not only do obese patients with different types of cancers have a worse prognosis but also they have an increased risk of metastatic disease and a shorter remission period after treatment, perhaps because angiogenesis may be exacerbated in obesity [104]. In angiogenesis, there is an equilibrium between angiogenic factors, which stimulate the proliferation and migration of endothelial cells, and antiangiogenic factors, which inhibit these activities. Endothelial cells abound in adipose tissue, and they secrete angiogenic factors, such as VEGF and HGF, which have autocrine/paracrine actions and endocrine effects elsewhere. Antiangiogenic factors such as angiostatin, endostatin, and adiponectin are also expressed in adipose tissue [82]. An altered balance between vascular growth factors and inhibitors is typical of obesity, with expansion of the capillary bed in regional fat depots [105], thus possibly contributing to the increased risk of metastatic disease in obese subjects with cancer.

5.10. Obesity-Induced Hypoxia. Oxygen levels in the WAT of obese mice are considerably lower than those of lean mice [106], and adipose tissue hypoxia may contribute to cancer risk. In particular, transcription factor HIF-1 $\alpha$ is correlated with increased metastatic spread and poor prognosis [107]. Studies from Bedogni et al. have previously demonstrated the importance of mild hypoxia of the skin in melanocyte transformation and the development of melanoma [108].

5.11. Oxidative Stress. Antioxidant activities may be independently decreased by obesity, thus increasing systemic oxidative stress, in part through the inflammatory process and in part independently of other mechanisms [109]. Increased oxidative damage to DNA may heighten cancer risk.

5.12. Shared Genetic Susceptibility. Genetic predisposition to obesity may predispose to the development of certain tumours, and it is now possible to identify in associated regions likely mutual candidate genes for cancer and obesity, independently of diabetes. Recently, a study in Scotland [110] reported that variants of common single nucleotide polymorphism around the melanocortin 4 receptor (MC4R) gene are associated with both obesity and colorectal cancer.

5.13. Endocrine Disruptors. Exposure to endocrine disruptors such as stress and food contaminants, which have an effect on the regular activity of estrogens, androgens, and insulin may also affect the simultaneous development of obesity and cancer [111].

5.14. Energy Homeostasis and Cancer. Coupled to insulin signalling, nutrient sensing pathways have been hypothesized 
to play a role in carcinogenesis. Several studies are currently examining targets such as AMP-activated protein kinase (AMPK), mammalian target of rapamycin (mTOR), fatty acid synthase, deacetylase SIRT1 (sirtuin 1), and epigenetic modulators [1].

5.15. Obesity, Immune Function and Risk of Cancer. Immune system alterations may be important in the higher incidence rates of cancer in obese patients since obesity has been correlated to a reduced immunocompetence in humans. Moulin et al. have recently shown that severely obese patients have a significantly lower natural killer (NK) cell cytotoxic activity as compared to normal individuals matched for age and gender, with lower defence against invaders such as precancerous and cancerous cells [112]. Interestingly, weight loss (26\% less of initial weight) is responsible for an increase in NK cytotoxic activity after 6 months from gastric bypass surgery [112]. Lynch et al. have recently shown that omental invariant NKT (iNKT) cell frequencies were lower in patients with severe obesity as compared to lean healthy subjects, thus suggesting a novel role for the omentum in immune regulation and tumor immunity [113].

The following list shows the possible factors involved in the development of cancer in obesity.

Anthropometric parameters:

body mass index (BMI) [3],

weight increase [26, 27],

visceral fat [18-23].

Lifestyle factors:

hypercaloric diet $[33,100]$,

high intake of animal fat, trans-fatty acids, and refined carbohydrates and low intake of fiber, vegetables, fruit, whole grain carbohydrates, and 25-hydroxyvitamin D (25(OH)D) [34, 37, 38],

sedentary lifestyle $[39,40]$.

Biological mechanisms:

hyperinsulinemia and insulin resistance [35, 41-45, $51,52]$

IGF-I, IGF-II, and IGF binding proteins [35],

sex hormones and sex hormone binding globulin [60-67],

chronic whole body low-grade inflammation [96100],

adipose tissue inflammation [70],

adipose tissue adipokine production (leptin, adiponectin, resistin, PAI-1, growth-related oncogenes, etc.) [71-78, 80-91, 94, 95],

adipose tissue vascular growth factors [82, 104],

shared genetic susceptibility [110],

oxidative stress [109],

endocrine disruptors [111],

immune function (NK cells and invariant NKT cells) $[112,113]$.

\section{Conclusions}

Obesity is responsible for approximately $20 \%$ of all malignancies, although its influence is gender and site specific. The association between obesity and a higher cancer risk is mainly due to anthropometric parameters and lifestyle factors which activate different biological mechanisms. Anthropometric parameters are BMI, weight increase, and the amount of body fat, particularly visceral fat. Lifestyle factors include sedentary habits and diet parameters, such as a hypercaloric and/or low-quality diet. The most important biological mechanisms mediating the unfavourable influence of the above factors are hyperinsulinemia and insulin resistance, the activities of IGFs and IGF binding proteins, sex hormones and SHBG, general and adipose tissue low-grade inflammation, changes in adipose tissue production of adipokines and vascular growth factors, oxidative stress, endocrine disruptors, and alterations in immune function. We do not yet have a clear scientific demonstration that avoiding increasing or reducing body weight significantly reduces the risk of cancer; however, the data from the surgical treatment of obese patients with a BMI higher than 40.0 are leading in this direction.

\section{Acknowledgments}

This work was supported by a Grant (IG11647, 2011) from the Italian Association for Cancer Research (AIRC), the Italian Ministry of University and Scientific Research (MIUR), PRIN 2009 , and local funds (e.g., $60 \%$ of University of Bari).

\section{References}

[1] E. E. Calle, C. Rodriguez, K. Walker-Thurmond, and M. J. Thun, "Overweight, obesity, and mortality from cancer in a prospectively studied cohort of U.S. adults," The New England Journal of Medicine, vol. 348, no. 17, pp. 1625-1638, 2003.

[2] E. E. Calle and R. Kaaks, "Overweight, obesity and cancer: epidemiological evidence and proposed mechanisms," Nature Reviews Cancer, vol. 4, no. 8, pp. 579-591, 2004.

[3] A. G. Renehan, M. Tyson, M. Egger, R. F. Heller, and M. Zwahlen, "Body-mass index and incidence of cancer: a systematic review and meta-analysis of prospective observational studies," The Lancet, vol. 371, no. 9612, pp. 569-578, 2008.

[4] World Cancer Research Fund, Food, Nutrition, Physical Activity, and the Prevention of Cancer: a Global Perspective, American Institute for Cancer Research, Washington, DC, USA, 2nd edition, 2007.

[5] K. Y. Wolin, K. Carson, and G. A. Colditz, "Obesity and cancer," Oncologist, vol. 15, no. 6, pp. 556-565, 2010.

[6] G. K. Reeves, K. Pirie, V. Beral, J. Green, E. Spencer, and D. Bull, "Cancer incidence and mortality in relation to body mass index in the million women study: Cohort study," British Medical Journal, vol. 335, no. 7630, pp. 1134-1139, 2007.

[7] International Agency for Research on Cancer, "Weight control and physical activity," in IARC Handbook of Cancer Prevention, H. Vainio and F. Bianchini, Eds., vol. 6, pp. 1-315, IARC Press, Lyon, France, 2002.

[8] C. M. Kitahara, E. A. Platz, L. E. B. Freeman et al., "Obesity and thyroid cancer risk among U.S. men and women: a pooled analysis of five prospective studies," Cancer Epidemiology Biomarkers and Prevention, vol. 20, no. 3, pp. 464-472, 2011. 
[9] M. A. Lichtman, "Obesity and the risk for a hematological malignancy: leukemia, lymphoma, or myeloma," Oncologist, vol. 15, no. 10, pp. 1083-1101, 2010.

[10] P. C. Enzinger and R. J. Mayer, "Esophageal cancer," The New England Journal of Medicine, vol. 349, no. 23, pp. 2241-2252, 2003.

[11] B. C. Jacobson, S. C. Somers, C. S. Fuchs, C. P. Kelly, and C. A. Camargo Jr., "Body-mass index and symptoms of gastroesophageal reflux in women," The New England Journal of Medicine, vol. 354, no. 22, pp. 2340-2348, 2006.

[12] J. Ahn, A. Schatzkin, J. V. Lacey Jr. et al., "Adiposity, adult weight change, and postmenopausal breast cancer risk," Archives of Internal Medicine, vol. 167, no. 19, pp. 2091-2102, 2007.

[13] S. E. Hankinson, G. A. Colditz, D. J. Hunter et al., "Reproductive factors and family history of breast cancer in relation to plasma estrogen and prolactin levels in postmenopausal women in the nurses' health study (United States)," Cancer Causes and Control, vol. 6, no. 3, pp. 217-224, 1995.

[14] L. Sjöström, A. Gummesson, C. D. Sjöström et al., "Effects of bariatric surgery on cancer incidence in obese patients in Sweden (Swedish Obese Subjects Study): a prospective, controlled intervention trial," The Lancet Oncology, vol. 10, no. 7, pp. 653662, 2009.

[15] M. Jeffreys, G. D. Smith, R. M. Martin, S. Frankel, and D. Gunnell, "Childhood body mass index and later cancer risk: a 50year follow-up of the Boyd Orr study," International Journal of Cancer, vol. 112, no. 2, pp. 348-351, 2004.

[16] T. Bjørge, A. Engeland, A. Tverdal, and G. D. Smith, "Body mass index in adolescence in relation to cause-specific mortality: a follow-up of 230,000 Norwegian adolescents," American Journal of Epidemiology, vol. 168, no. 1, pp. 30-37, 2008.

[17] A. G. Renehan, I. Soerjomataram, M. Tyson et al., "Incident cancer burden attributable to excess body mass index in 30 European countries," International Journal of Cancer, vol. 126, no. 3, pp. 692-702, 2010.

[18] M. Harvie, L. Hooper, and A. H. Howell, "Central obesity and breast cancer risk: a systematic review," Obesity Reviews, vol. 4, no. 3, pp. 157-173, 2003.

[19] A. A. Arslan, K. J. Helzlsouer, C. Kooperberg et al., "Anthropometry, physical activity, and the risk of pancreatic cancer in the European prospective investigation into cancer and nutrition," JAMA Internal Medicine, vol. 170, no. 9, pp. 791-802, 2010.

[20] C. Friedenreich, A. Cust, P. H. Lahmann et al., "Anthropometric factors and risk of endometrial cancer: the European prospective investigation into cancer and nutrition," Cancer Causes and Control, vol. 18, no. 4, pp. 399-413, 2007.

[21] Z. Dai, Y.-C. Xu, and L. Niu, "Obesity and colorectal cancer risk: a meta-analysis of cohort studies," World Journal of Gastroenterology, vol. 13, no. 31, pp. 4199-4206, 2007.

[22] A. A. Moghaddam, M. Woodward, and R. Huxley, "Obesity and risk of colorectal cancer: a meta-analysis of 31 studies with 70,000 events," Cancer Epidemiology Biomarkers and Prevention, vol. 16, no. 12, pp. 2533-2547, 2007.

[23] A. Steffen, M. B. Schulze, T. Pischon et al., "Anthropometry and esophageal cancer risk in the European prospective investigation into cancer and nutrition," Cancer Epidemiology Biomarkers and Prevention, vol. 18, no. 7, pp. 2079-2089, 2009.

[24] K. A. Britton, J. M. Massaro, J. M. Murabito, B. E. Kreger, U. Hoffmann, and C. S. Fox, "Body fat distribution, incident cardiovascular disease, cancer, and all-cause mortality," Journal of the American College of Cardiology, 2013.
[25] C. Zhang, K. M. Rexrode, R. M. Van Dam, T. Y. Li, and F. B. Hu, "Abdominal obesity and the risk of all-cause, cardiovascular, and cancer mortality: sixteen years of follow-up in US women," Circulation, vol. 117, no. 13, pp. 1658-1667, 2008.

[26] P. T. Campbell, M. Cotterchio, E. Dicks, P. Parfrey, S. Gallinger, and J. R. McLaughlin, "Excess body weight and colorectal cancer risk in Canada: associations in subgroups of clinically defined familial risk of cancer," Cancer Epidemiology Biomarkers and Prevention, vol. 16, no. 9, pp. 1735-1744, 2007.

[27] S. Hosono, K. Matsuo, K. Hirose et al., "Weight gain during adulthood and body weight at age 20 are associated with the risk of endometrial cancer in japanese women," Journal of Epidemiology, vol. 21, no. 6, pp. 466-473, 2011.

[28] S. Pendyala, L. M. Neff, M. Suárez-Fariñas, and P. R. Holt, "Diet-induced weight loss reduces colorectal inflammation: implications for colorectal carcinogenesis," American Journal of Clinical Nutrition, vol. 93, no. 2, pp. 234-242, 2011.

[29] J. R. Jaggers, X. Sui, S. P. Hooker et al., "Metabolic syndrome and risk of cancer mortality in men," European Journal of Cancer, vol. 45, no. 10, pp. 1831-1838, 2009.

[30] J. R. Daling, K. E. Malone, D. R. Doody, L. G. Johnson, J. R. Gralow, and P. L. Porter, "Relation of body mass index to tumor markers and survival among young women with invasive ductal breast carcinoma," Cancer, vol. 92, no. 4, pp. 720-729, 2001.

[31] J. J. Griggs, M. E. S. Sorbero, and G. H. Lyman, "Undertreatment of obese women receiving breast cancer chemotherapy," Archives of Internal Medicine, vol. 165, no. 11, pp. 1267-1273, 2005.

[32] Y. Chen, X. Wang, J. Wang, Z. Yan, and J. Luo, "Excess body weight and the risk of primary liver cancer: an updated metaanalysis of prospective studies," European Journal of Cancer, vol. 48, no. 14, pp. 2137-2145, 2012.

[33] S. R. Spindler, "Rapid and reversible induction of the longevity, anticancer and genomic effects of caloric restriction," Mechanisms of Ageing and Development, vol. 126, no. 9, pp. 960-966, 2005.

[34] D. C. McMillan, N. Sattar, M. Lean, and C. S. McArdle, "Obesity and cancer," British Medical Journal, vol. 333, no. 7578, pp. 11091111, 2006.

[35] R. Pallavi, M. Giorgio, and P. G. Pelicci, "Insights into the beneficial effect of caloric/dietary restriction for a healthy and prolonged life," Frontiers in Physiology, vol. 3, no. 318, pp. 1-10, 2012.

[36] I. Imayama, C. M. Ulrich, C. M. Alfano et al., "Effects of a caloric restriction weight loss diet and exercise on inflammatory biomarkers in overweight/obese postmenopausal women: a randomized controlled trial," Cancer Research, vol. 72, no. 9, pp. 2314-2326, 2012.

[37] E. J. Fine, C. J. Segal-Isaacson, R. D. Feinman et al., “Targeting insulin inhibition as a metabolic therapy in advanced cancer: a pilot safety and feasibility dietary trial in 10 patients," Nutrition, vol. 28, no. 10, pp. 1028-1035, 2012.

[38] Z. Lagunova, A. C. Porojnicu, W. B. Grant, Ø. Bruland, and J. E. Moan, "Obesity and increased risk of cancer: does decrease of serum 25-hydroxyvitamin D level with increasing body mass index explain some of the association?" Molecular Nutrition and Food Research, vol. 54, no. 8, pp. 1127-1133, 2010.

[39] A. McTiernan, "Mechanisms linking physical activity with cancer," Nature Reviews Cancer, vol. 8, no. 3, pp. 205-211, 2008.

[40] L. H. Kushi, C. Doyle, M. McCullough et al., "American Cancer Society guidelines on nutrition and physical activity for cancer prevention: reducing the risk of cancer with healthy food 
choices and physical activity," CA: Cancer Journal for Clinicians, vol. 62 , no. 1, pp. 30-67, 2012.

[41] S. Tsugane and M. Inoue, "Insulin resistance and cancer: epidemiological evidence," Cancer Science, vol. 101, no. 5, pp. 10731079, 2010.

[42] M. J. Gunter, D. R. Hoover, H. Yu et al., "Insulin, insulin-like growth factor-I, endogenous estradiol, and risk of colorectal cancer in postmenopausal women," Cancer Research, vol. 68, no. 1, pp. 329-337, 2008.

[43] M. J. Gunter, D. R. Hoover, H. Yu et al., "Insulin, insulin-like growth factor-I, and risk of breast cancer in post-menopausal women," Journal of the National Cancer Institute, vol. 101, no. 1, pp. 48-60, 2009.

[44] G. C. Kabat, M. Kim, B. J. Caan et al., "Repeated measures of serum glucose and insulin in relation to postmenopausal breast cancer," International Journal of Cancer, vol. 125, no. 11, pp. 2704-2710, 2009.

[45] M. J. Gunter, D. R. Hoover, H. Yu et al., "A prospective evaluation of insulin and insulin-like growth factor-I as risk factors for endometrial cancer," Cancer Epidemiology Biomarkers and Prevention, vol. 17, no. 4, pp. 921-929, 2008.

[46] S. Becker, L. Dossus, and R. Kaaks, "Obesity related hyperinsulinaemia and hyperglycaemia and cancer development," Archives of Physiology and Biochemistry, vol. 115, no. 2, pp. 86-96, 2009.

[47] P. Pisani, "Hyper-insulinaemia and cancer, meta-analyses of epidemiological studies," Archives of Physiology and Biochemistry, vol. 114, no. 1, pp. 63-70, 2008.

[48] A. E. Cust, N. E. Allen, S. Rinaldi et al., "Serum levels of C-peptide, IGFBP-1 and IGFBP-2 and endometrial cancer risk; results from the European prospective investigation into cancer and nutrition," International Journal of Cancer, vol. 120, no. 12, pp. 2656-2664, 2007.

[49] M. Verheus, P. H. M. Peeters, S. Rinaldi et al., "Serum C-peptide levels and breast cancer risk: results from the European prospective investigation into cancer and nutrition (EPIC)," International Journal of Cancer, vol. 119, no. 3, pp. 659-667, 2006.

[50] J. Ma, H. Li, E. Giovannucci et al., "Prediagnostic body-mass index, plasma C-peptide concentration, and prostate cancerspecific mortality in men with prostate cancer: a long-term survival analysis," The Lancet Oncology, vol. 9, no. 11, pp. 1039-1047, 2008.

[51] F. Frasca, G. Pandini, L. Sciacca et al., "The role of insulin receptors and IGF-I receptors in cancer and other diseases," Archives of Physiology and Biochemistry, vol. 114, no. 1, pp. 23-37, 2008.

[52] M. E. Cox, M. E. Gleave, M. Zakikhani et al., "Insulin receptor expression by human prostate cancers," Prostate, vol. 69, no. 1, pp. 33-40, 2009.

[53] B. T.-Y. Chan and A. V. Lee, "Insulin receptor substrates (IRSs) and breast tumorigenesis," Journal of Mammary Gland Biology and Neoplasia, vol. 13, no. 4, pp. 415-422, 2008.

[54] E. J. Gallagher and D. LeRoith, "The proliferating role of insulin and insulin-like growth factors in cancer," Trends in Endocrinology and Metabolism, vol. 21, no. 10, pp. 610-618, 2010.

[55] A. G. Renehan, J. Frystyk, and A. Flyvbjerg, "Obesity and cancer risk: the role of the insulin-IGF axis," Trends in Endocrinology and Metabolism, vol. 17, no. 8, pp. 328-336, 2006.

[56] L. Héron-Milhavet and D. LeRoith, "Insulin-like growth factor I induces MDM2-dependent degradation of p53 via the p38 MAPK pathway in response to DNA damage," Journal of Biological Chemistry, vol. 277, no. 18, pp. 15600-15606, 2002.
[57] Y. Wu, S. Yakar, L. Zhao, L. Hennighausen, and D. LeRoith, "Circulating insulin-like growth factor-I levels regulate colon cancer growth and metastasis," Cancer Research, vol. 62, no. 4, pp. 1030-1035, 2002.

[58] A. Canonici, W. Steelant, V. Rigot et al., "Insulin-like growth factor-I receptor, E-cadherin and $\alpha \mathrm{v}$ integrin form a dynamic complex under the control of $\alpha$-catenin," International Journal of Cancer, vol. 122, no. 3, pp. 572-582, 2008.

[59] G. De Pergola, M. Zamboni, N. Pannacciulli et al., "Divergent effects of short-term very low calorie diet (VLCD) on insulinlike growth factror-I (IGF-I) and insulin-like growth factor binding protein-3 (IGFBP-3) serum concentrations in premenopausal obese women," Obesity Research, vol. 6, no. 6, pp. 408415, 1998.

[60] R. Kaaks, S. Rinaldi, T. J. Key et al., "Postmenopausal serum androgens, oestrogens and breast cancer risk: the European prospective investigation into cancer and nutrition," EndocrineRelated Cancer, vol. 12, no. 4, pp. 1071-1082, 2005.

[61] K. A. Brown and E. R. Simpson, "Obesity and breast cancer: progress to understanding the relationship," Cancer Research, vol. 70, no. 1, pp. 4-7, 2010.

[62] R. Kaaks, F. Berrino, T. Key et al., "Serum sex steroids in premenopausal women and breast cancer risk within the European prospective investigation into cancer and nutrition (EPIC)," Journal of the National Cancer Institute, vol. 97, no. 10, pp. 755$765,2005$.

[63] N. E. Allen, T. J. Key, L. Dossus et al., "Endogenous sex hormones and endometrial cancer risk in women in the European prospective investigation into cancer and nutrition (EPIC)," Endocrine-Related Cancer, vol. 15, no. 2, pp. 485-497, 2008.

[64] R. C. Travis and T. J. Key, "Oestrogen exposure and breast cancer risk," Breast Cancer Research, vol. 5, no. 5, pp. 239-247, 2003.

[65] A. V. Lee, J. G. Jackson, J. L. Gooch et al., "Enhancement of insulin-like growth factor signaling in human breast cancer: estrogen regulation of insulin receptor substrate-1 expression in vitro and in vivo," Molecular Endocrinology, vol. 13, no. 5, pp. 787-796, 1999.

[66] R. Kaaks, A. Lukanova, and M. S. Kurzer, "Obesity, endogenous hormones, and endometrial cancer risk: a synthetic review," Cancer Epidemiology Biomarkers and Prevention, vol. 11, no. 12, pp. 1531-1543, 2002.

[67] C. A. Derby, S. Zilber, D. Brambilla, K. H. Morales, and J. B. McKinlay, "Body mass index, waist circumference and waist to hip ratio and change in sex steroid hormones: the Massachusetts male ageing study," Clinical Endocrinology, vol. 65, no. 1, pp. 125131, 2006.

[68] Y. Matsuzawa, "Therapy insight: adipocytokines in metabolic syndrome and related cardiovascular disease," Nature Clinical Practice Cardiovascular Medicine, vol. 3, no. 1, pp. 35-42, 2006.

[69] M. J. Khandekar, P. Cohen, and B. M. Spiegelman, "Molecular mechanisms of cancer development in obesity," Nature Reviews Cancer, vol. 11, no. 12, pp. 886-895, 2011.

[70] T. H. Mayi, M. Daoudi, B. Derudas et al., "Human adipose tissue macrophages display activation of cancer-related pathways," Journal of Biological Chemistry, vol. 287, pp. 21904-21913, 2012.

[71] L. Vona-Davis and D. P. Rose, "Adipokines as endocrine, paracrine, and autocrine factors in breast cancer risk and progression," Endocrine-Related Cancer, vol. 14, no. 2, pp. 189-206, 2007.

[72] S. Catalano, S. Marsico, C. Giordano et al., "Leptin enhances, via AP-1, expression of aromatase in the MCF-7 cell line," Journal of Biological Chemistry, vol. 278, no. 31, pp. 28668-28676, 2003. 
[73] X. Hu, S. C. Juneja, N. J. Maihle, and M. P. Cleary, "Leptin a growth factor in normal and malignant breast cells and for normal mammary gland development," Journal of the National Cancer Institute, vol. 94, no. 22, pp. 1704-1711, 2002.

[74] M. P. Cleary, F. C. Phillips, S. C. Getzin et al., "Genetically obese MMTV-TGF- $\alpha$ /Lepob Lepob female mice do not develop mammary tumors," Breast Cancer Research and Treatment, vol. 77, no. 3, pp. 205-215, 2003.

[75] N. Stefanou, V. Papanikolaou, Y. Furukawa, Y. Nakamura, and A. Tsezou, "Leptin as a critical regulator of hepatocellular carcinoma development through modulation of human telomerase reverse transcriptase," BMC Cancer, vol. 10, article 442, 2010.

[76] M.-N. Dieudonne, F. Machinal-Quelin, V. Serazin-Leroy, M.C. Leneveu, R. Pecquery, and Y. Giudicelli, "Leptin mediates a proliferative response in human MCF7 breast cancer cells," Biochemical and Biophysical Research Communications, vol. 293, no. 1, pp. 622-628, 2002.

[77] I. Barone, S. Catalano, L. Gelsomino et al., "Leptin mediates tumor-stromal interactions that promote the invasive growth of breast cancer cells," Cancer Research, vol. 72, no. 6, pp. 1416-1427, 2012.

[78] M. Cnop, P. J. Havel, K. M. Utzschneider et al., "Relationship of adiponectin to body fat distribution, insulin sensitivity and plasma lipoproteins: evidence for independent roles of age and sex," Diabetologia, vol. 46, no. 4, pp. 459-469, 2003.

[79] M. Fasshauer, J. Klein, S. Neumann, M. Eszlinger, and R. Paschke, "Hormonal regulation of adiponectin gene expression in 3T3-L1 adipocytes," Biochemical and Biophysical Research Communications, vol. 290, no. 3, pp. 1084-1089, 2002.

[80] M.-N. Dieudonne, M. Bussiere, E. Dos Santos, M.-C. Leneveu, Y. Giudicelli, and R. Pecquery, "Adiponectin mediates antiproliferative and apoptotic responses in human MCF7 breast cancer cells," Biochemical and Biophysical Research Communications, vol. 345, no. 1, pp. 271-279, 2006.

[81] E. Brakenhielm, N. Veitonmaki, R. Cao et al., "Adiponectininduced antiangiogenesis and antitumor activity involve caspase-mediated endothelial cell apoptosis," Proceedings of the National Academy of Sciences of USA, vol. 101, no. 8, pp. 24762481, 2004.

[82] G. J. Byrne, A. Ghellal, J. Iddon et al., "Serum soluble vascular cell adhesion molecule-1: role as a surrogate marker of angiogenesis," Journal of the National Cancer Institute, vol. 92, no. 16, pp. 1329-1336, 2000.

[83] Y. Wang, K. S. Lam, and A. Xu, "Adiponectin as a negative regulator in obesity-related mammary carcinogenesis," Cell Research, vol. 17, no. 4, pp. 280-282, 2007.

[84] Y. Miyoshi, T. Funahashi, S. Kihara et al., "Association of serum adiponectin levels with breast cancer risk," Clinical Cancer Research, vol. 9, no. 15, pp. 5699-5704, 2003.

[85] C. Mantzoros, E. Petridou, N. Dessypris et al., "Adiponectin and breast cancer risk," Journal of Clinical Endocrinology and Metabolism, vol. 89, no. 3, pp. 1102-1107, 2004.

[86] S. S. Tworoger, A. H. Eliassen, T. Kelesidis et al., "Plasma adiponectin concentrations and risk of incident breast cancer," Journal of Clinical Endocrinology and Metabolism, vol. 92, no. 4, pp. 1510-1516, 2007.

[87] L. Dal Maso, L. S. Augustin, A. Karalis et al., "Circulating adiponectin and endometrial cancer risk," Journal of Clinical Endocrinology and Metabolism, vol. 89, no. 3, pp. 1160-1163, 2004.

[88] A. E. Cust, R. Kaaks, C. Friedenreich et al., "Plasma adiponectin levels and endometrial cancer risk in pre- and post-menopausal women," Journal of Clinical Endocrinology and Metabolism, vol. 92, no. 1, pp. 255-263, 2007.

[89] E. K. Wei, E. Giovannucci, C. S. Fuchs, W. C. Willett, and C. S. Mantzoros, "Low plasma adiponectin levels and risk of colorectal cancer in men: a prospective study," Journal of the National Cancer Institute, vol. 97, no. 22, pp. 1688-1694, 2005.

[90] C. Grommes, G. E. Landreth, and M. T. Heneka, "Antineoplastic effects of peroxisome proliferator-activated receptor $\gamma$ agonists," Lancet Oncology, vol. 5, no. 7, pp. 419-429, 2004.

[91] J. C. Carter and F. C. Church, "Obesity and breast cancer: the roles of peroxisome proliferator-activated receptor- $\gamma$ and plasminogen activator inhibitor-1," PPAR Research, vol. 2009, Article ID 345320, 13 pages, 2009.

[92] E. Maury, S. M. Brichard, Z. Pataky, A. Carpentier, A. Golay, and E. Bobbioni-Harsch, "Effect of obesity on growth-related oncogene factor- $\alpha$, thrombopoietin, and tissue inhibitor metalloproteinase-1 serum levels," Obesity, vol. 18, no. 8, pp. 1503$1509,2010$.

[93] B. R. Lane, J. Liu, P. J. Bock et al., "Interleukin-8 and growth-regulated oncogene alpha mediate angiogenesis in Kaposi's sarcoma," Journal of Virology, vol. 76, no. 22, pp. 11570-11583, 2002.

[94] H. Haghnegahdar, J. Du, D. Wang et al., "The tumorigenic and angiogenic effects of MGSA/GRO proteins in melanoma," Journal of Leukocyte Biology, vol. 67, no. 1, pp. 53-62, 2000.

[95] E. Lupia, O. Bosco, S. Bergerone et al., "Thrombopoietin contributes to enhanced platelet activation in patients with unstable angina," Journal of the American College of Cardiology, vol. 48, no. 11, pp. 2195-2203, 2006.

[96] M. L. Slattery, K. Curtin, E. M. Poole et al., "Genetic variation in C-reactive protein in relation to colon and rectal cancer risk and survival," International Journal of Cancer, vol. 128, no. 11, pp. 2726-2734, 2011.

[97] L. Dossus, S. Rinaldi, S. Becker et al., "Obesity, inflammatory markers, and endometrial cancer risk: a prospective case-control study," Endocrine-Related Cancer, vol. 17, no. 4, pp. 10071019, 2010.

[98] A. Mantovani, P. Allavena, A. Sica, and F. Balkwill, "Cancerrelated inflammation," Nature, vol. 454, no. 7203, pp. 436-444, 2008.

[99] R. C. M. Van Kruijsdijk, E. Van Der Wall, and F. L. J. Visseren, "Obesity and cancer: the role of dysfunctional adipose tissue," Cancer Epidemiology Biomarkers and Prevention, vol. 18, no. 10, pp. 2569-2578, 2009.

[100] J. Ye and J. N. Keller, "Regulation of energy metabolism by inflammation: a feedback response in obesity and calorie restriction," Aging, vol. 2, no. 6, pp. 361-368, 2010.

[101] M. S. Rodeheffer, K. Birsoy, and J. M. Friedman, "Identification of white adipocyte progenitor cells In vivo," Cell, vol. 135, no. 2, pp. 240-249, 2008.

[102] B. Dirat, L. Bochet, G. Escourrou, P. Valet, and C. Muller, "Unraveling the obesity and breast cancer links: a role for cancer-associated adipocytes?" Endocrine Development, vol. 19, pp. 45-52, 2010.

[103] C. F. Bellows, Y. Zhang, J. Chen, M. L. Frazier, and M. G. Kolonin, "Circulation of progenitor cells in obese and lean colorectal cancer patients," Cancer Epidemiology Biomarkers and Prevention, vol. 20, no. 11, pp. 2461-2468, 2011.

[104] C. L. Amling, R. H. Riffenburgh, L. Sun et al., "Pathologic variables and recurrence rates as related to obesity and race in men with prostate cancer undergoing radical prostatectomy," Journal of Clinical Oncology, vol. 22, no. 3, pp. 439-445, 2004. 
[105] J. V. Silha, M. Krsek, P. Sucharda, and L. J. Murphy, "Angiogenic factors are elevated in overweight and obese individuals," International Journal of Obesity, vol. 29, no. 11, pp. 1308-1314, 2005.

[106] J. Ye, Z. Gao, J. Yin, and Q. He, "Hypoxia is a potential risk factor for chronic inflammation and adiponectin reduction in adipose tissue of ob/ob and dietary obese mice," American Journal of Physiology, vol. 293, no. 4, pp. E1118-E1128, 2007.

[107] P. Vaupel and M. Hoeckel, "Predictive power of the tumor oxygenation status," Advances in Experimental Medicine and Biology, vol. 471, pp. 533-539, 1999.

[108] B. Bedogni, S. M. Welford, A. C. Kwan, J. Ranger-Moore, K. Saboda, and M. B. Powell, "Inhibition of phosphatidylinositol3 -kinase and mitogen-activated protein kinase kinase $1 / 2$ prevents melanoma development and promotes melanoma regression in the transgenic TPRas mouse model," Molecular Cancer Therapeutics, vol. 5, no. 12, pp. 3071-3077, 2006.

[109] A. B. Crujeiras, A. Díaz-Lagares, M.C. Carreira, M. Amil, and F. F. Casanueva, "Oxidative stress associated to dysfunctional adipose tissue: a potential link between obesity, type 2 diabetes mellitus and breast cancer," Free Radical Research, vol. 47, no. 4, pp. 243-256, 2013.

[110] A. Tenesa, H. Campbell, E. Theodoratou et al., "Common genetic variants at the MC4R locus are associated with obesity, but not with dietary energy intake or colorectal cancer in the Scottish population," International Journal of Obesity, vol. 33, no. 2, pp. 284-288, 2009.

[111] J. Legler, T. Hamers, M. Van Eck van der Sluijs-van de Bor et al., "The OBELIX project: early life exposure to endocrine disruptors and obesity," The American Journal of Clinical Nutrition, vol. 94, supplement 6, pp. 1933S-1938S, 2009.

[112] C. M. Moulin, L. V. Rizzo, and A. Halpern, "Effect of surgeryinduced weight loss on immune function," Expert Review of Gastroenterology and Hepatology, vol. 2, no. 5, pp. 617-619, 2008.

[113] L. Lynch, D. O'Shea, D. C. Winter, J. Geoghegan, D. G. Doherty, and C. O'Farrelly, "Invariant NKT cells and CD1d+ cells amass in human omentum and are depleted in patients with cancer and obesity," European Journal of Immunology, vol. 39, no. 7, pp. 1893-1901, 2009. 


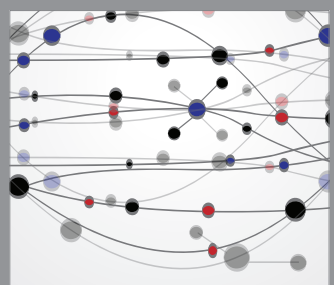

The Scientific World Journal
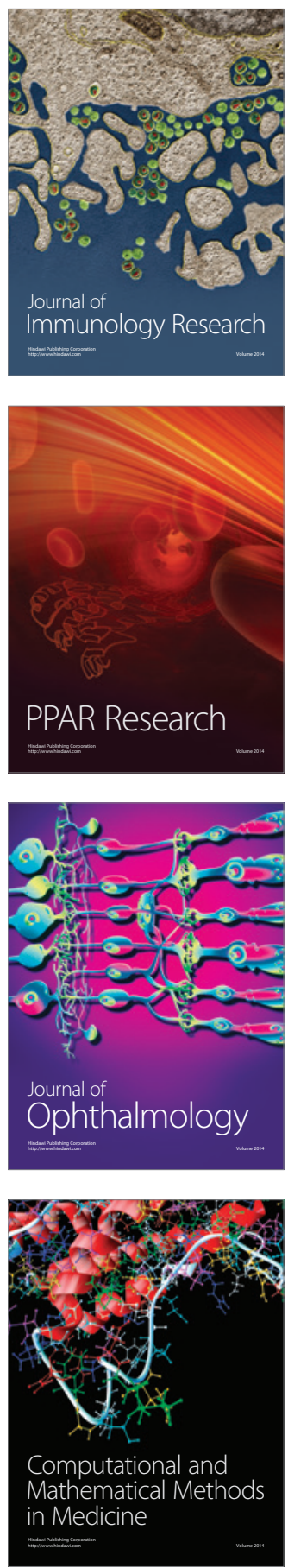

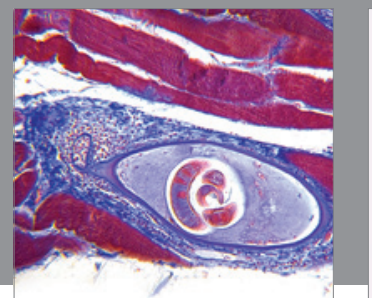

Gastroenterology

Research and Practice
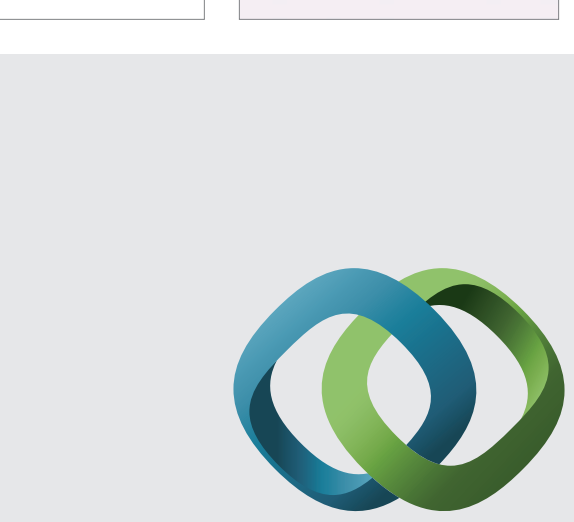

\section{Hindawi}

Submit your manuscripts at

http://www.hindawi.com
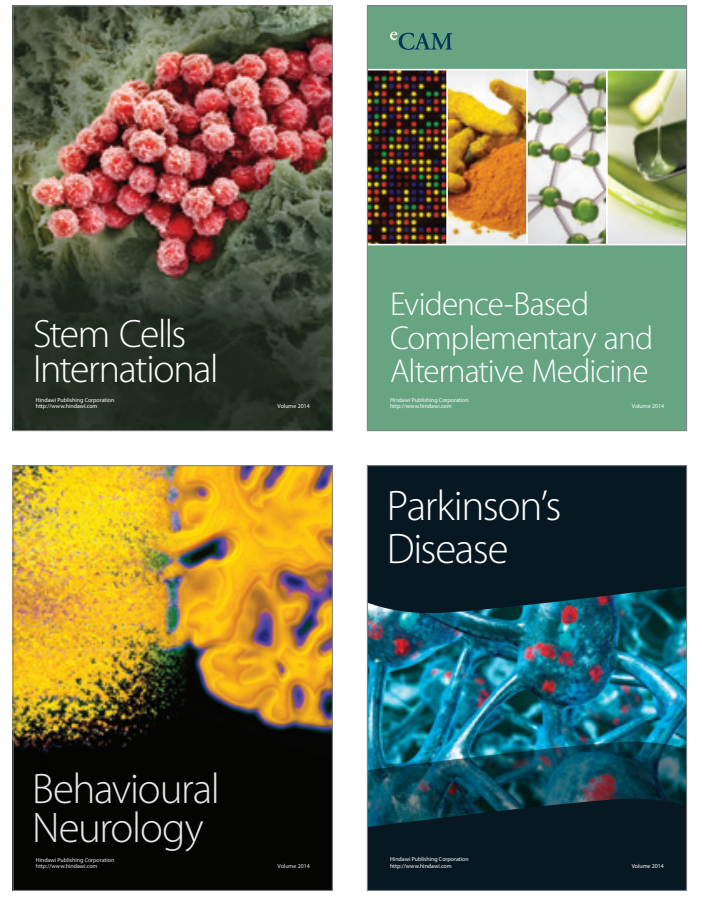
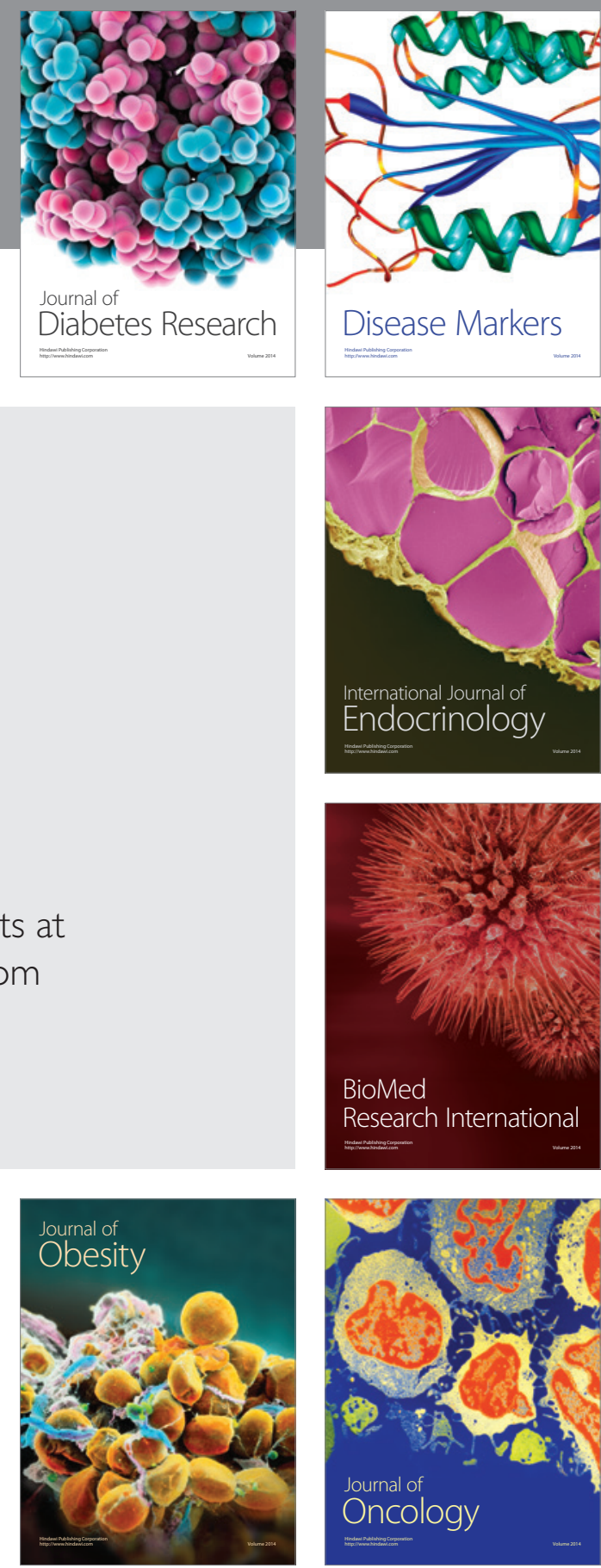

Disease Markers
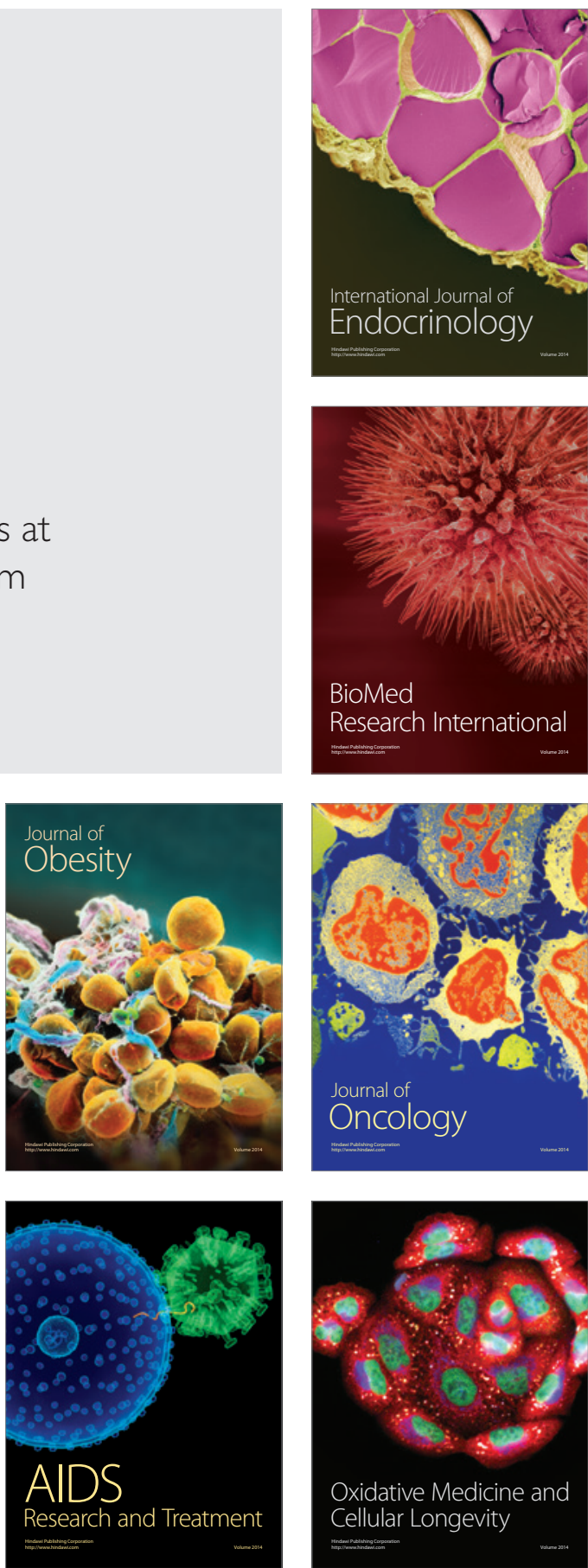